Article

\title{
Numerical Investigation of Periodic Fluctuations in Energy Efficiency in Centrifugal Pumps at Different Working Points
}

\author{
Hehui Zhang ${ }^{1}$, Shengxiang Deng ${ }^{1, *}$ and Yingjie $\mathrm{Qu}^{2}$ \\ 1 School of Energy Science and Engineering, Central South University, Changsha 410083, China; \\ hehuizhang@csu.edu.cn \\ 2 Hunan M \&W Energy Saving Tech. Co., Ltd., Changsha 410208, China; pump118@163.com \\ * Correspondence: csdsx@163.com; Tel.: +86-731-8887-9863
}

Academic Editor: Bjørn H. Hjertager

Received: 10 December 2016; Accepted: 2 March 2017; Published: 10 March 2017

\begin{abstract}
In order to simulate the energy efficiency fluctuation behavior of an industrial centrifugal pump with a six-blade impeller, a full-scale three-dimensional (3D) an unsteady state computational fluid dynamics (CFD) model was used. Five operational points with different flow fluxes were numerically investigated by using the Navier-Stokes code with shear-stress transport (SST) k- $\omega$ turbulence model. The predicted performance curves agreed well with the test data. A sine function was fitted to the transient calculation results and the results show that the efficiency fluctuates mainly on the blade passing frequency, while the fluctuation level varies with flow rate. Furthermore, high efficiency is not necessarily associated with low fluctuation level. The efficiency fluctuation level is high at part-load points, and becomes relatively low when flow rate exceeds the design value. The effect of change in torque is greater than that of the head lift with respect to fluctuations of efficiency. Based upon the analysis of velocity vector distribution of different impeller phase positions, a hypothesis which considers both the effect of pump's structural shape and flow fluxes was proposed to explain the above behavior by analyzing the impeller-tongue interaction. This work enriches the theoretical system of flow parameters fluctuation of centrifugal pump, and provides useful insight for the optimal design of centrifugal pumps.
\end{abstract}

Keywords: centrifugal pump; energy efficiency; computational fluid dynamics; fluctuation

\section{Introduction}

The centrifugal pump is a kind of energy conversion device widely used in various process industries. However, it consumes large amounts of energy for fluid transportation and pressure enhancement, making energy efficiency one of its most important performance indicators [1]. A great deal of research has been done on centrifugal pumps for improving their efficiency under rated conditions, and for expanding the range of the high-efficiency region. Research has mainly focused on the optimization of inner flow field in centrifugal pumps. Generally, computational fluid dynamics (CFD) methods are used for flow field analysis and prediction of performance of centrifugal pumps due to their low cost, quick development speed and convenience for comparing different design schemes [2-4]. According to a number of published reports, flow field structures and performance curves predicted by CFD methods are the predominant basis for optimization of pump product design to obtain higher efficiencies [5-8]. For example, Shi et al. [8] numerically investigated the quantitative relationship between efficiency and impeller design parameters of a forward-extended double-blade sewage pump, and obtained a response surface of the highest efficiency under different design factors 
through regression analysis. In their work, the change in efficiency predicted by CFD calculations under all factors and levels set by orthogonal table was less than 5\% [8].

Generally, most CFD research is aimed at improving the efficiency of the centrifugal pump and is based on steady state models, in which the impeller region with a fixed phase position is set in rotating reference frame to simulate its rotation. In this case, the possibility of a change in efficiency under different impeller phase positions is completely neglected. In fact, the inside flow field fluctuation of centrifugal pump is obvious due to the interaction between impeller blade and volute tongue, which has been popular field of research in recent years. Flow field information such as secondary flow structure changes and pressure fluctuations are obtained through unsteady CFD calculations, while sliding mesh technique for the impeller region is used to reflect the real dynamic effect of the rotating impeller. As early as 2002, González et al. [9] systematically studied the dynamic effects of the impeller-volute interaction of a centrifugal pump on the inside flow by using 3D transient flow numerical simulations, which was focused on the change in flow pattern with different impeller circumferential positions on both design and off-design points. On each operational point, the pressure inside the volute fluctuated mainly at the blade passing frequency, whereas the fluctuation amplitude and irregularity were larger on the off-design point than those on the design point, which led to corresponding force changes on the main shaft of the pump. Also in 2002, Treutz [10] performed transient flow analysis in centrifugal pumps with commercial CFD code CFX, and validated the computation results with experimental measurements including pump characteristics and local pressure fluctuation in the volute casing. Treutz's work focused more on the development of a transient simulation method and the detailed analysis of the secondary flow field itself, but seldom involved the energy efficiency fluctuation which is likely to be caused by the interaction between volute casing and the blades within it. In 2003, Guo et al. [11] experimentally studied the fluctuation behavior of fluid forces on shaft, and reported that there was no necessary correlation for fluctuation amplitude between pressure and fluid forces. Later, around the inside flow field fluctuation caused by the impeller-volute interaction, more experimental and numerical studies were undertaken to describe the transient flow phenomena and explain the formation mechanism behind it (e.g., Cheah et al. [12] in 2008, Lucius et al. [13] in 2010, Huang et al. [14] and Dai et al. [15] in 2013). The unsteady state analysis on fluctuation of flow parameters, such as pressure and hydraulic forces, can provide an essential reference for blade design [16,17], flow rate setup [18,19] and impact assessment under special working conditions [20].

The hydraulic efficiency of centrifugal pump merely depends on the values of shaft torque and head lift under constant flow rate and rotation speed, while these parameters are controlled by fluid forces and pressure. Since there is obvious fluctuation of fluid forces and pressure, it is obvious that the efficiency will also show some periodic trends of fluctuation. However, compared with the considerable research on the fluctuation of fluid forces and pressure, few reports are found on the fluctuation of efficiency. Thus, the major objective in this article is to fill the gap in research on the efficiency fluctuation behavior of centrifugal pumps. A full-scale 3D numerical simulation of unsteady flow was carried out to study an industrial centrifugal pump with six blades, and the time-varying flow parameters were recorded to investigate the efficiency fluctuation behavior at five different operational points.

\section{Numerical Procedure}

\subsection{Pump Geometry and Design Parameters}

An industrial horizontal double-entry split centrifugal pump with six blades (manufactured by Hunan M \& W Energy Saving Tech. Co. Ltd., Changsha, China) was studied in this work. The pump was designed to work with clean water with the highest temperature of $65^{\circ} \mathrm{C}$. The inner hydraulic region of pump was modeled to be a set of three different parts: a suction chamber, an extruding chamber and the impeller between the above two chambers. The 3D geometry of the hydraulic model 
is shown in Figure 1, while the main structure and performance parameters of the pump are listed in Table 1. Both the suction and exit centers are located at the same horizontal level.

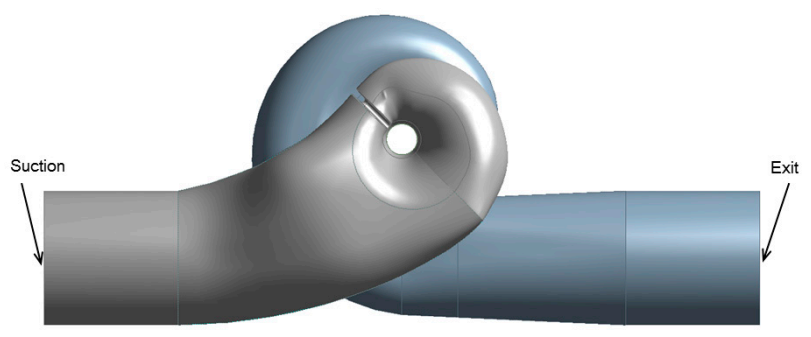

Figure 1. 3D geometry of inner fluid region of the pump.

Table 1. Important design parameters of the pump studied in this work.

\begin{tabular}{cc}
\hline Structural Parameter & Value \\
\hline Suction diameter & $D_{s}=0.35 \mathrm{~m}$ \\
Exit diameter & $D_{e}=0.35 \mathrm{~m}$ \\
Impeller inlet diameter & $d_{1}=0.08 \mathrm{~m}$ \\
Impeller outlet diameter & $d_{2}=0.29 \mathrm{~m}$ \\
Impeller outlet width & $b_{2}=0.136 \mathrm{~m}$ \\
Rated flow & $Q_{\text {des }}=0.35 \mathrm{~m}^{3} / \mathrm{s}$ \\
Rated head & $H_{\text {des }}=17 \mathrm{~m}$ \\
Rotation speed & $n=1480 \mathrm{rpm}$ \\
NPSHr & $\Delta h_{r}=3.2 \mathrm{~m}$ \\
\hline
\end{tabular}

To better understand the inner structure of the volute and impeller, a middle section of the volute fluid region and impeller are illustrated in Figure 2. The plane shown in Figure 2 is perpendicular to the main shaft of the pump. An X-Y rectangular coordinate system is given on the plane, where the original point of the coordinate system and the center point of the impeller coincide with each other. Both the volute tongue and exit face are in the fourth quadrant of the coordinate system. As shown in Figure 2, the impeller rotates anticlockwise, and the rotary phase is set to be the zero phase position when the impeller center, one blade tip and the volute tongue vertex are located on the same straight line.

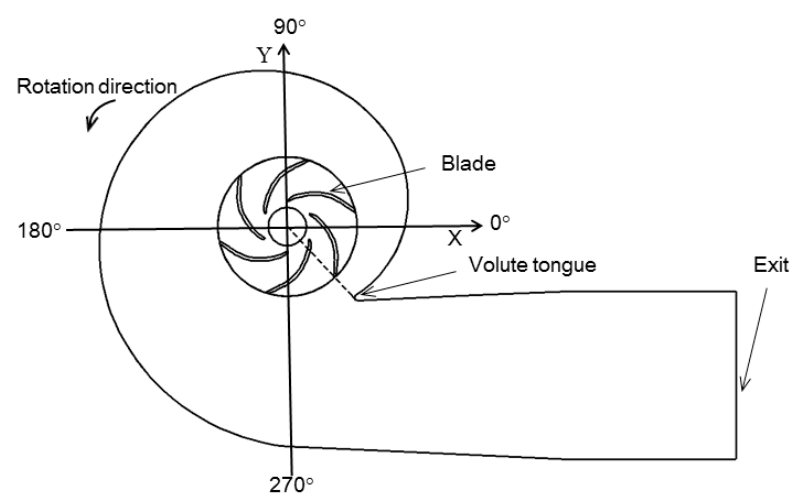

Figure 2. Mid-plane view of the inner fluid region of the pump.

\subsection{Numerical Model and Sliding Technology}

The commercial CFD software Fluent 14.0 was used to perform the calculations for 3D full-scale model by solving Reynolds averaged incompressible Navier-Stokes equations based on finite volume method. The mass and momentum conservation equations are given as:

$$
\frac{\partial \rho}{\partial t}+\nabla \cdot(\rho \vec{u})=0
$$




$$
\frac{\partial}{\partial t}(\rho \vec{u})+\nabla \cdot(\rho \vec{u} \vec{u})=-\nabla p+\nabla \cdot\left[\mu\left(\nabla \vec{u}+(\nabla \vec{u})^{T}\right)\right]+\rho \vec{g}+\vec{f}
$$

where $\vec{u}$ is the velocity vector $\left(\mathrm{m} \cdot \mathrm{s}^{-1}\right), t$ represents time $(\mathrm{s}), \rho$ is density $\left(\mathrm{kg} \cdot \mathrm{m}^{-3}\right), p$ is static pressure (Pa), $\vec{f}$ is external body force term $\left(\mathrm{N} \cdot \mathrm{m}^{-3}\right), \vec{g}$ is the gravity constant $\left(\mathrm{m} \cdot \mathrm{s}^{-2}\right)$ and $\mu$ is the effective viscosity $\left(\mathrm{kg} \cdot \mathrm{m}^{-1} \cdot \mathrm{s}^{-1}\right)$.

The full model was made up of two different kinds of zones: the stationary zone for the suction and extruding chambers, and the motion zone for the impeller region. In the stationary zone, for a general scalar, $\phi$, in an arbitrary control volume, $V\left(\mathrm{~m}^{3}\right)$, the convection-diffusion-reaction (CDR) equation in its integral form can be written as Equation (3):

$$
\frac{d}{d t} \int_{V} \rho \phi d V+\int_{\partial V} \rho \phi \vec{u} \cdot d \vec{A}=\int_{\partial V} \Gamma \nabla \phi \cdot d \vec{A}+\int_{V} S_{\phi} d V
$$

where $\vec{A}$ is the area vector of the faces on the control volume $\left(\mathrm{m}^{2}\right), \phi$ is the generic variable for solving the equation, and $\Gamma$ and $S_{\phi}$ are the diffusion coefficient and source term of $\phi$, respectively. In the equation of continuity, $\phi$ is set as 1 and $\Gamma$ is 0 . However, in the momentum equation, $\phi$ is the velocity component and $\Gamma$ is the dynamic viscosity $\left(\mathrm{kg} \cdot \mathrm{m}^{-1} \cdot \mathrm{s}^{-1}\right)$. From left to right, the four items in Equation (3) represent the mass variation over time, the convection term, the diffusion term and the source term, respectively.

For the motion zone, Fluent 14.0 provided a dynamic mesh model with sliding technology [21] to better reflect the real fluid flow under the impeller-tongue interaction. With this technology, all the nodes in impeller region rotate rigidly around the center, and the dynamic mesh zone gets connected to the stationary zone of extruding chamber through non-conformal interfaces. When the mesh rotation is updated each time, the non-conformal interfaces are also likewise updated, so the new phase difference between the impeller and tongue can be truly reflected. The general transport equation in Equation (3) also applies to the dynamic mesh model, except for the convection term, which is left out due to the consideration of the mesh's motion. Therefore, the conservation equation for the above general scalar, $\phi$, in the motion zone can be represented by Equation (4) [21].

$$
\frac{d}{d t} \int_{V} \rho \phi d V+\int_{\partial V} \rho \phi\left(\vec{u}-\vec{u}_{g}\right) \cdot d \vec{A}=\int_{\partial V} \Gamma \nabla \phi \cdot d \vec{A}+\int_{V} S_{\phi} d V
$$

where $\vec{u}_{g}$ is the mesh velocity vector of the moving mesh $\left(\mathrm{m} \cdot \mathrm{s}^{-1}\right)$.

As there is no shape change for all of the mesh cells during the rotation of impeller, the control volume $V$ remains constant, and therefore the time derivative term in Equation (4) can be simplified to Equation (5) by using first-order backward difference.

$$
\frac{d}{d t} \int_{V} \rho \phi d V=\frac{(\rho \phi V)_{n+1}-(\rho \phi V)_{n}}{\Delta t}
$$

where $n$ denotes the respective value at the nth time level, and $n+1$ represents its next time level.

For the turbulence model, the $k-\omega$-based shear-stress transport (SST) model developed by Menter [22] was used to solve the mass and momentum conservation equations. The $k$ - $\omega$-based SST model is especially well-suited for the turbulence problems occurring in high rotational speed turbomachinery due to its reasonable balance between the computational cost and accuracy. In the far field region with free shear flow, the standard $k-\varepsilon$ model is used to speed up the calculations, while in the near-wall region with intense separation flow induced by the multi-curvature revolving blade walls, the turbulence model is gradually transformed into $k-\omega$ model to ensure the resolution of the analysis. Details of the coefficients of turbulence model can be found in a previously published report [22]. 
The calculation procedure was separated into two stages. The first stage was for a steady model, while the second was for an unsteady model. There were five different cases to be computed in this study, while each one corresponded to a certain working condition. The flow rates for the five cases were $0.6 Q_{d e s}, 0.8 Q_{d e s}, 1.0 Q_{d e s}, 1.2 Q_{d e s}$ and $1.4 Q_{d e s}$, respectively. The steady model was computed in advance to validate the calculation results with test data and then provide the initial condition for subsequent transient numerical simulation, where the impeller was located on the zero phase position. In the steady model, the full fluid region consists of two different reference frames. These reference frames were the stationary frame for the suction and extruding chambers, and the rotating frame with a rotating speed of $1480 \mathrm{r} \cdot \mathrm{min}^{-1}$ for the impeller zone. A specified flow velocity was set on the suction face as the inlet boundary condition with flow direction perpendicular to the face, and the turbulence intensity was set at $5 \%$. An outflow condition was given on the exit face of the extruding chamber to capture the fully developed pressure and velocity distribution. All interior faces of the fluid region consisted of no-slip rough walls, and according to the specifications of the processing technology, the roughness of the blades and other faces were set to be $5 \times 10^{-5} \mathrm{~m}$ and $10^{-4} \mathrm{~m}$, respectively. The internal leakage of pump was ignored for simplification. Physical property parameters of pure water, including density and dynamic viscosity at $25^{\circ} \mathrm{C}$ were given as the fluid properties. The convergence criterion was less than $10^{-4}$ for mean residues of both mass and momentum. The whole fluid region was meshed with hexahedral structure elements to ensure convergence and computational speed. To run the grid sensitivity test, seven computational cases with different mesh sizes but fixed flow rate $1.0 Q_{\text {des }}$ were studied to obtain the changes in head and efficiency values. Both the predicted head and efficiency values become very stable after the fourth point (see Figure 3), whose rangeability becomes less than $0.3 \%$ and $0.2 \%$ respectively, so the mesh of that point was selected for computation in this work in order to save computation time. The mesh information including elements and nodes number was listed in Table 2, and the element quality, skewness factor and orthogonal quality of the mesh was $0.73,0.76$ and 0.81 respectively. The maximum non-dimensional wall distance $y+<60$ was obtained in the full flow field of the final computation results by performing the Yplus mesh adaption refinement function [21] during numerical computations.

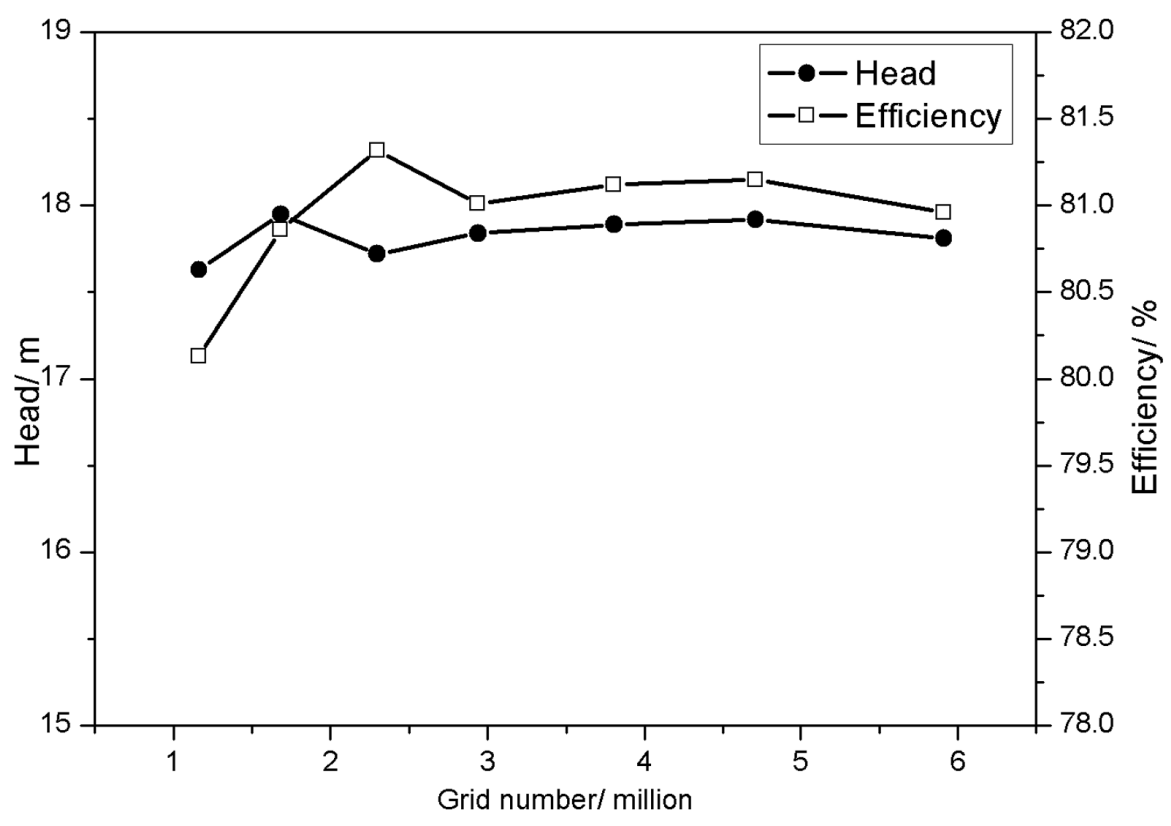

Figure 3. Predicted head values with different grid numbers. 
Table 2. Mesh information obtained in the current work.

\begin{tabular}{ccc}
\hline Fluid Zone & Number of Elements & Number of Nodes \\
\hline Suction chamber & 832,871 & $2,966,467$ \\
Extruding chamber & $1,105,902$ & $4,331,943$ \\
Impeller & 999,772 & $3,474,289$ \\
Total & $2,938,544$ & $10,772,699$ \\
\hline
\end{tabular}

\subsection{Transient Calculations}

After steady state computation, each case was transformed into a transient one with the steady result as its initial condition. There were mainly two differences between the unsteady and steady state models. First of all, the rotating reference frame for the impeller fluid was discarded, and a sliding mesh technique was used with a mesh motion speed of $1480 \mathrm{r} \cdot \mathrm{min}^{-1}$ to simulate the real fluid effect of the impeller's rotation. For the solution setup, the total calculation time was $0.2432 \mathrm{~s}$, corresponding to six shaft rotation cycles. The time step $\Delta \mathrm{t}$ was defined according to the Equation (6).

$$
\Delta t=\frac{T}{360}
$$

where $T$ is the rotation period of the main shaft and had the value of $0.04054 \mathrm{~s}$. This means that the impeller fluid region meshes rotate $1^{\circ}$ anticlockwise for each time step. The unsteady flow calculation results of the last shaft rotation cycle were chosen for analysis when the transient iteration was fully convergent and the fluctuation in flow parameters was regular. The head lift and fluid torque on impeller were monitored during the whole computational process, and the energy efficiency for each time step was calculated using Equation (7).

$$
\eta=\frac{955 \rho g Q H}{n N}
$$

where $g$ is the gravitational acceleration $\left(9.8 \mathrm{~N} \cdot \mathrm{kg}^{-1}\right), Q$ is the volume flow rate $\left(\mathrm{m}^{3} \cdot \mathrm{s}^{-1}\right), H$ is the head of the pump $(\mathrm{m}), n$ is the shaft's rotational speed $\left(\mathrm{rev} \cdot \mathrm{min}^{-1}\right)$, and $N$ is the torque $(\mathrm{N} \cdot \mathrm{m})$ on the impeller. The pump head $H$ can be obtained using Equation (8).

$$
H=\frac{P_{o}-P_{i}}{\rho g}
$$

where $P_{o}$ and $P_{i}$ represent the total pressure of the exit and entrance faces of the pump, respectively.

\section{Numerical Data Processing and Validation}

\subsection{Regression Analysis}

Regression analysis was performed to quantitatively research the efficiency of the fluctuation behavior. For each working condition with a fixed flow rate, it is obvious from Equation (5) that all influencing factors on efficiency are constants except for the torque $N$ and pump head $H$. According to the published experimental and simulation reports on the flow field parameters [7,8], a sine function was chosen for regression analysis of the fluctuation tendency of the torque, head and finally the efficiency in the transient calculation results. The sine function for fitting the above three flow parameters can be expressed using Equation (9).

$$
f(t)=B \sin (\omega t+\varphi)+C
$$

where $B$ and $C$ are peak value and displacement distance on vertical coordinate, respectively, and share the same units with the fitting object. Furthermore, $\omega$ is the angular rotational rate of the shaft $\left(\mathrm{rad} \cdot \mathrm{s}^{-1}\right)$, $t$ is the time (s), and $\varphi$ is the initial phase of sine function (rad). 


\subsection{Curve Comparison after Normalization}

The main aim of the regression analysis is to describe the periodic fluctuation characters quantitatively, especially to reflect the amplitude of the fluctuation. As represented by Equation (5), there are three flow parameters, namely the efficiency and its influencing factors torque and head, which will be studied by regression analysis. However, it is impossible to apply comparative study on these three flow parameters because they have different dimensions and value ranges. Thus, the normalization process was carried out to make the value ranges of different parameters less than 1 . This way, it will become convenient to compare the fluctuation amplitude of different flow parameters at different working points, and hence, identify the underlying leading factors contributing to the fluctuation in the efficiency.

A method named maximum normalization was chosen to normalize the values. The method makes linear transformation of the original data according to Equation (10).

$$
f(t)^{*}=\frac{f(t)}{f(t)_{\max }}
$$

where $f(t)$ is the original sine function for fitting, and $f(t)^{*}$ is its value after normalization. Furthermore, $f(t)_{\max }$ is the maximum value $(A+B)$ of the sine function. In this way, the peak value reflecting fluctuation amplitude is turned into a dimensionless normalized form $A /(A+B)$, which allows comparison among different flow parameters after the regression analysis.

\subsection{Test Validation}

To ensure the correctness of the CFD results, a test on a full-size pump was made according to Grade 2 of the ISO 9906-2012 standard [23], as shown in Figure 4. The pumped medium was cold clean water. Two pressure gauges with an accuracy class of 0.4 were mounted on the pipe near the pump flange, with one upstream of the entrance face while the other was downstream of the exit face. An electromagnetic flowmeter was installed on the straight pipe, and was used to measure the water flow velocity, which can be multiplied by the pipe sectional area to obtain the volumetric flow rate. A torque-meter and a laser tachometer (each having 0.2 accuracy class) were applied to record the motor torque and rotation speed, respectively.

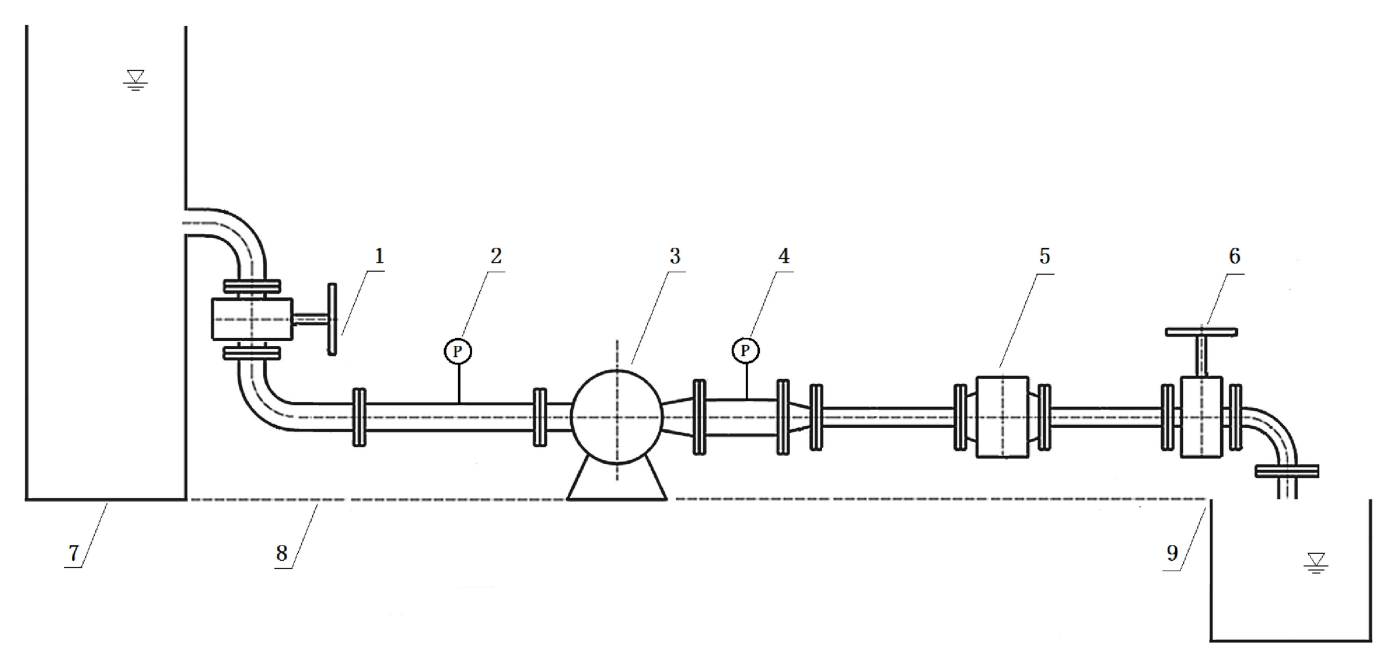

Figure 4. Schematic diagram of the test rig. 1: inlet valve; 2 : vacuum gauge; 3 : centrifugal pump driven by motor; 4: pressure gauge; 5 : electromagnetic flowmeter; 6 : outlet valve; 7: upstream tank; 8: mounting datum; 9: downstream tank. 


\section{Results and Discussion}

\subsection{Performance Curve Verification}

At first, the pump performance curves including flow head curve $(Q \sim H)$ and flow efficiency curve $(Q \sim \eta)$ were drawn in Figure 5 to compare the calculated results with the test data. Beside the results from steady state model, the average head and efficiency values during the last shaft rotating cycle in the unsteady state model were also included in the comparison. Generally, the performance curves predicted by the CFD agreed well with the test data, and the small difference between them might have been caused by the computational and test random errors, processing deviations of flow-passing surfaces of the test pump, and the CFD model simplification. Besides, there is also mechanical power loss in experiment due to engineering factors in practice, such as the rotation of coupling, friction within bearings, friction between bearing and shaft, and friction between the sealing ring and shaft. Although the power loss due to internal fluid leakage was ignored in computation model, the CFD calculation efficiency values might still be lower than the test ones when the power loss due to friction exceeds the loss caused by internal leakage. This phenomenon can be found in Figure $5 \mathrm{~b}$. On the point with flow flux of $1.0 Q_{\text {des }}$, the highest energy efficiency is reached, which conforms to the design specification. Calculation results from steady and transient models have the same trend, though the predicted head and efficiency values of the transient model are closer to the experimental results than those obtained from the steady state model for most of the operating points. A preliminary explanation is that the steady state model can only represent the pump performance on a certain impeller phase position, while the mean values of the transient state model reduce the deviation of fluctuation by considering all the impeller phase positions.

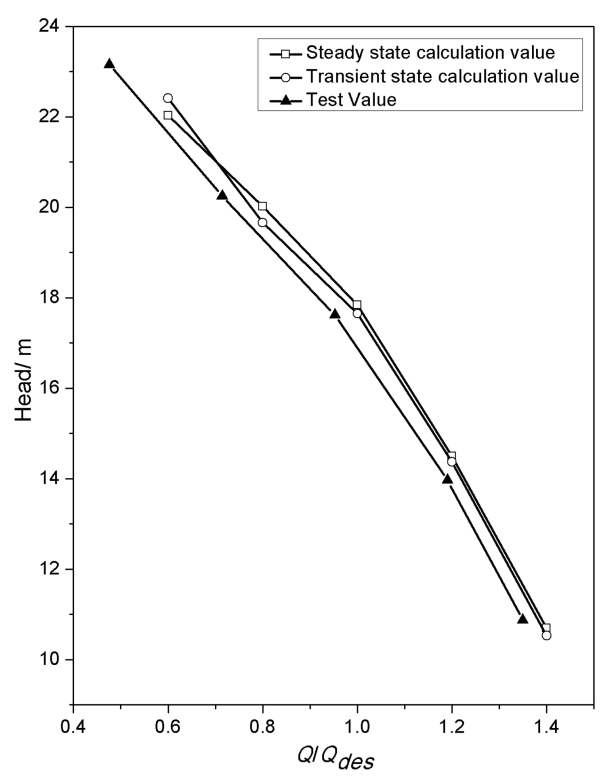

(a)

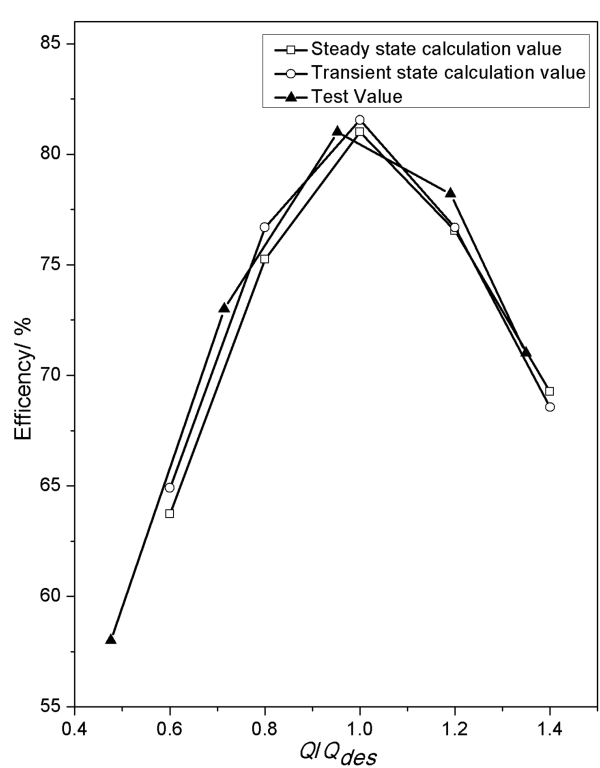

(b)

Figure 5. Comparison between the simulated and experimental results: (a) flow-head curve $(Q \sim H)$; (b) flow-efficiency curve $(Q \sim \eta)$.

\subsection{Periodic Fluctuation Behavior}

A detailed quantitative analysis can be made based upon the transient CFD results to discover the fluctuation behavior of the flow field. In the last shaft rotation cycle, the time-variation of the flow field parameters, including torque, head and finally efficiency, for different working conditions are shown in Figures 6-8, respectively. The red dots in these figures represent the calculated values for each time step. To better understand the fluctuation trend, the discrete time series values were fitted 
using the sine function. The fitting parameters are listed in Tables $3-5$, and the fitted curves were drawn using black solid lines as shown in Figures 6-8. The fitting optimization index $R^{2}$ for all the curves exceeded 0.8 , showing a very good fit. Since the efficiency values do not come directly from the CFD results, but from the calculation of the torque and head, the $R^{2}$ values are slightly lower for them.

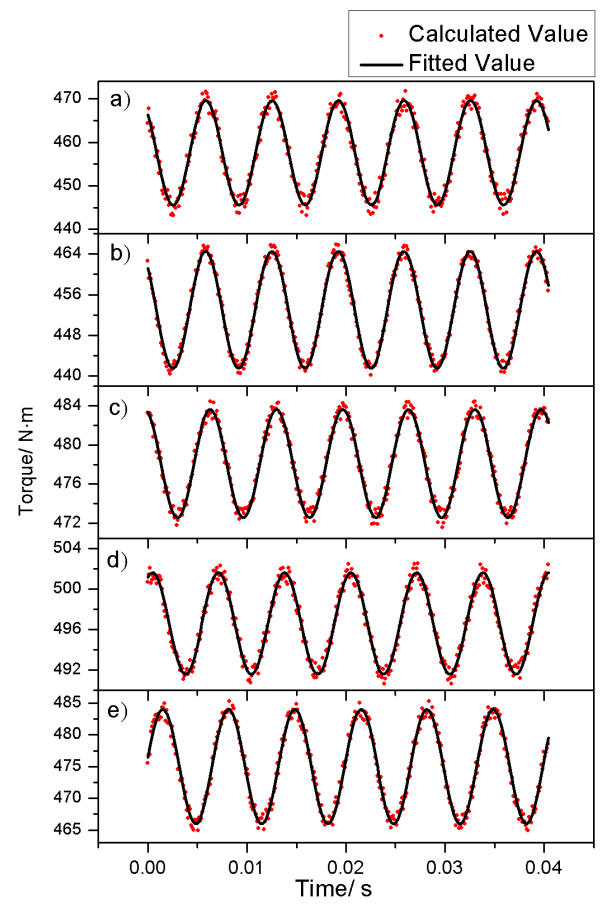

Figure 6. Fluctuation of torque in one shaft rotation cycle on different working points: (a) $0.6 Q_{\text {des }}$; (b) $0.8 Q_{\text {des }} ;$ (c) $1.0 Q_{\text {des }} ;$ (d) $1.2 Q_{\text {des }} ;$ (e) $1.4 Q_{\text {des }}$.

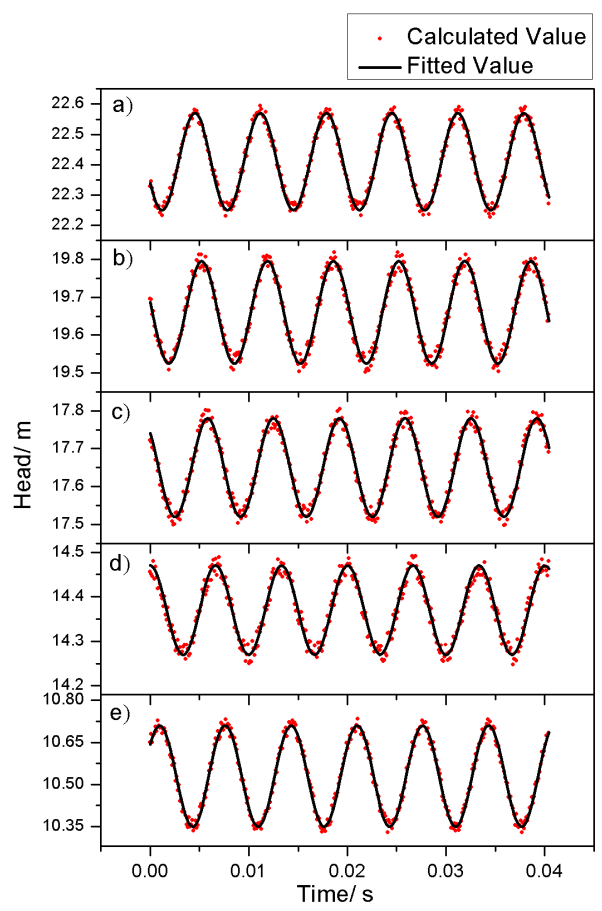

Figure 7. Fluctuation of head in one shaft rotation cycle at different working points: (a) $0.6 Q_{d e s}$; (b) $0.8 Q_{\text {des }} ;$ (c) $1.0 Q_{\text {des }} ;$ (d) $1.2 Q_{\text {des }} ;$ (e) $1.4 Q_{\text {des }}$. 


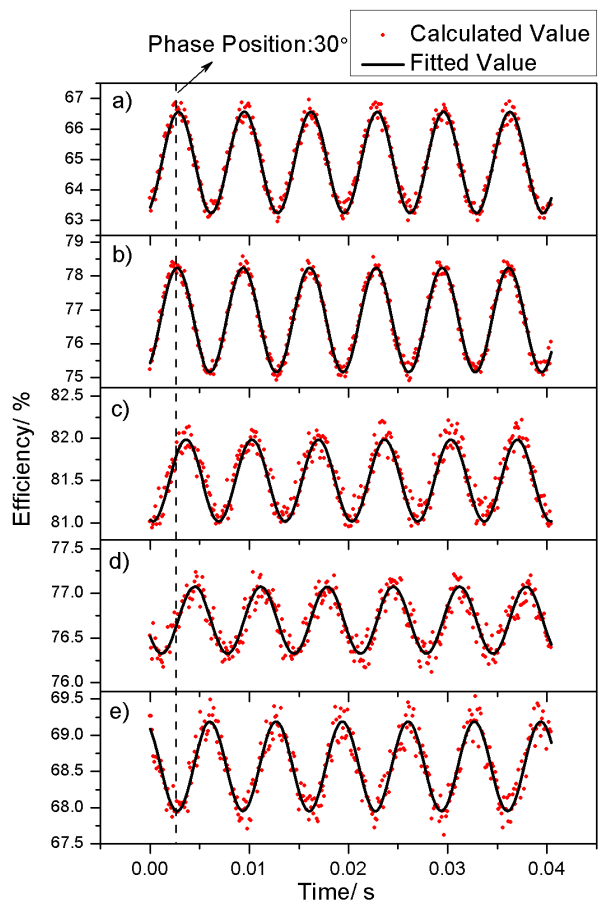

Figure 8. Fluctuation of efficiency in one shaft rotation cycle at different working points: (a) $0.6 Q_{\text {des }}$; (b) $0.8 Q_{\text {des }} ;$ (c) $1.0 Q_{\text {des }} ;$ (d) $1.2 Q_{\text {des }} ;$ (e) $1.4 Q_{\text {des }}$.

Table 3. Fitting parameters for torque fluctuation in different working conditions. B: peak value on the vertical coordinate; $C$ : displacement distance on the vertical coordinate; $\omega$ : angular rotational rate of the shaft $\left(\mathrm{rad} \cdot \mathrm{s}^{-1}\right) ; \phi$ : initial phase of sine function (rad); $R^{2}$ : fitting optimization index.

\begin{tabular}{cccccc}
\hline & $\mathbf{0 . 6} Q_{\text {des }}$ & $\mathbf{0 . 8} Q_{\text {des }}$ & $\mathbf{1 . 0} Q_{\text {des }}$ & $\mathbf{1 . 2} Q_{\text {des }}$ & $\mathbf{1 . 4 Q _ { \text { des } }}$ \\
\hline$B$ & 12.10 & 11.50 & 5.51 & 5.04 & 8.96 \\
$\omega$ & 940.1 & 942.0 & 940.1 & 940.1 & 940.1 \\
$\phi$ & 2.33 & 2.34 & 1.93 & 1.15 & 0.18 \\
$C$ & 457.5 & 453.0 & 478.0 & 497.0 & 475.0 \\
$R^{2}$ & 0.93 & 0.92 & 0.90 & 0.94 & 0.91 \\
\hline
\end{tabular}

Table 4. Fitting parameters for head fluctuation in different working conditions.

\begin{tabular}{cccccc}
\hline & $\mathbf{0 . 6} \boldsymbol{Q}_{\text {des }}$ & $\mathbf{0 . 8} \boldsymbol{Q}_{\text {des }}$ & $\mathbf{1 . 0} \boldsymbol{Q}_{\text {des }}$ & $\mathbf{1 . 2} \boldsymbol{Q}_{\text {des }}$ & $\mathbf{1 . 4 Q _ { \text { des } }}$ \\
\hline$B$ & 0.159 & 0.135 & 0.130 & 0.101 & 0.180 \\
$\omega$ & 942.9 & 951.5 & 940.1 & 940.1 & 940.1 \\
$\phi$ & -2.70 & 2.95 & 2.37 & 1.63 & 0.66 \\
$C$ & 23.4 & 20.1 & 17.7 & 15.2 & 11.5 \\
$R^{2}$ & 0.92 & 0.95 & 0.94 & 0.91 & 0.92 \\
\hline
\end{tabular}

Table 5. Fitting parameters for efficiency fluctuation in different working conditions.

\begin{tabular}{cccccc}
\hline & $\mathbf{0 . 6} Q_{\text {des }}$ & $\mathbf{0 . 8} Q_{\text {des }}$ & $\mathbf{1 . 0} Q_{\text {des }}$ & $\mathbf{1 . 2} Q_{\text {des }}$ & $\mathbf{1 . 4 Q _ { \text { des } }}$ \\
\hline$B$ & 1.66 & 1.54 & 0.484 & 0.374 & 0.617 \\
$\omega$ & 940.1 & 940.1 & 940.1 & 940.1 & 943.0 \\
$\phi$ & -1.09 & -0.97 & -1.81 & -2.64 & 2.16 \\
$C$ & 64.9 & 76.7 & 81.5 & 76.7 & 68.6 \\
$R^{2}$ & 0.91 & 0.92 & 0.89 & 0.84 & 0.88 \\
\hline
\end{tabular}


Three main findings can be obtained from Figures 6-8. Firstly, the sine function periods of all curves are equal to one-sixth-fold of the shaft rotation cycle. Since it triggers obvious second flow when each blade passes near the tongue, the dominating fluctuation frequency of flow field parameters is usually the blade passing frequency (BPF). This phenomenon has been validated by many researchers [4,7-9]. Although all curves share the same period time, their initial phase values are different. Furthermore, there is no unified law for the initial phase for each flow parameter on each operational point. This is due to the initial phase value of flow parameters, which depends on the detailed flow field distribution including both velocity and pressure at the start of each shaft rotation cycle. Lastly but most importantly, there are huge differences between the peak values of each flow field parameter among different working conditions.

The peak values listed in Table 5 show that for the pump studied in this work, the maximum fluctuation value of efficiency is as high as $1.66 \%$, while the minimum value exceeds $0.37 \%$. The fluctuation level basically has the same magnitude as the reported increased efficiency value based on steady state CFD models (e.g., Shi et al. [8], Kim et al. [24] and Zhao et al. [25]). Therefore, it is preferable to take the unsteady state CFD model to optimize the design of the centrifugal pump which can improve its determinacy.

The peak value can provide the most intuitive information about the fluctuation behavior. The comparison of dimensionless wave peak values on different working conditions for torque, head and efficiency are shown in Figure 9. As listed in Tables 3-5, the original peak values vary considerably for different parameters, however their normalized quantities are in the same order of magnitude, thus providing a common comparison basis. Unlike the simple intuition, low flow parameter fluctuation lever does not necessarily associate with the high energy efficiency. Instead, the minimum normalized wave peak values of all three parameters appear on the operating point with a $1.2 Q_{d e s}$ flow flux. Furthermore, all of these values clearly rise when the flow flux exceeds $1.2 Q_{d e s}$. Generally, the torque and efficiency curves (shown in Figure 9) share similar trends. On the part-load points, the normalized peak values for the above two parameters are very high, and they both reach the maximum values on the point with $0.6 Q_{d e s}$ flow flux.

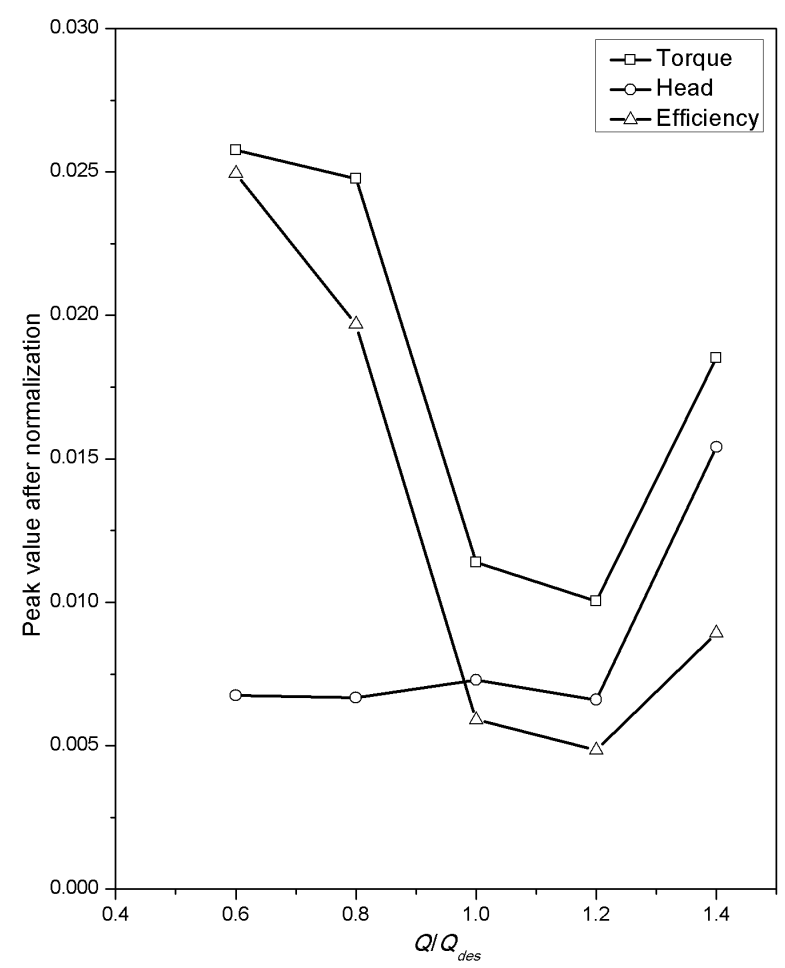

Figure 9. Dimensionless peak values after the process of normalization. $Q$ : volume flow rate $\left(\mathrm{m}^{3} \cdot \mathrm{s}^{-1}\right)$. 
The curve of the normalized peak value of the head has completely different characteristics from the other two curves (as shown in Figure 9). This is because there is little change before the flow flux reaches the value of $1.2 Q_{\text {des }}$. Thus, it can be speculated that there is a high degree of correlation between the fluctuation of torque and efficiency, especially for the part load working points. Further research is still needed to discover the detailed mechanism of the fluctuation of efficiency. However, the current findings suggest that the toque fluctuation might be the leading factor (among others) which contributes to the fluctuation of efficiency.

\subsection{Flow Structures Analysis}

It can be said superficially that the fluctuation in the pump efficiency is caused by the periodic behavior of the macroscopic flow parameters, such as torque and head. However, the actual root cause can only be revealed through the structural analysis of the flow field. To do so, the operating points including $0.6 Q_{d e s}, 1.0 Q_{d e s}$, and $1.4 Q_{\text {des }}$ were chosen for further study, and the velocity vector distributions on the mid-plane are shown in Figures 10-12, respectively. In each figure, a rectangular area near the tongue with the size of $0.25 \mathrm{~m} \times 0.20 \mathrm{~m}$ is picked out and magnified for better observation, which is marked with black lines. For each working point, the flow fields on the phase positions of both $0^{\circ}$ and $30^{\circ}$ are shown for comparison. Note that the time corresponds to the phase position of $30^{\circ}$ is marked as a vertical dash line in Figure 8. Therefore, the efficiency value in that position can be observed clearly. In addition, the initial point with a $0^{\circ}$ phase is also shown in the figure.

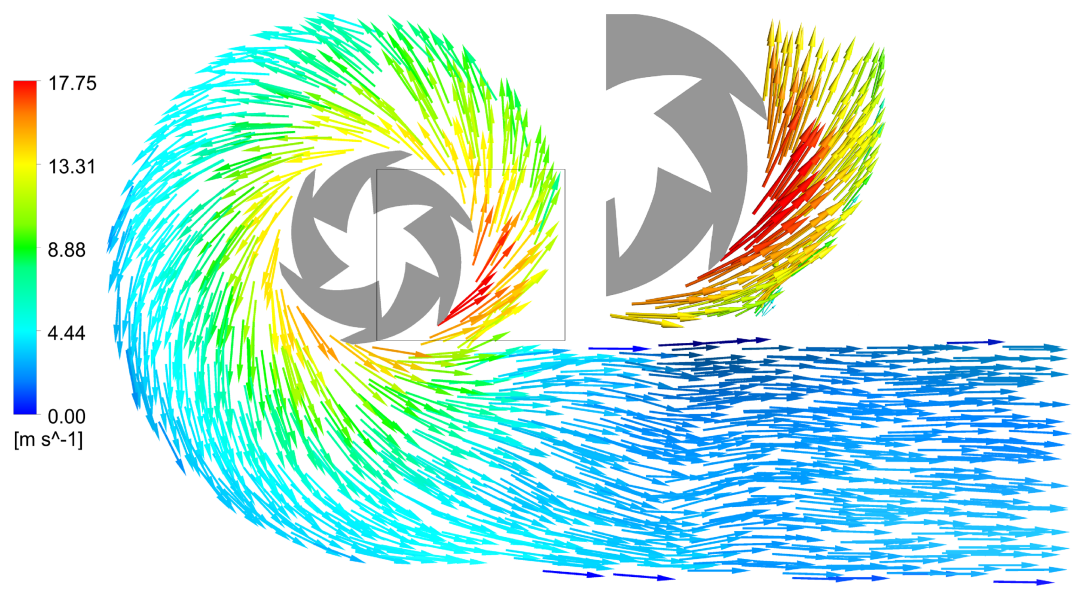

(a)

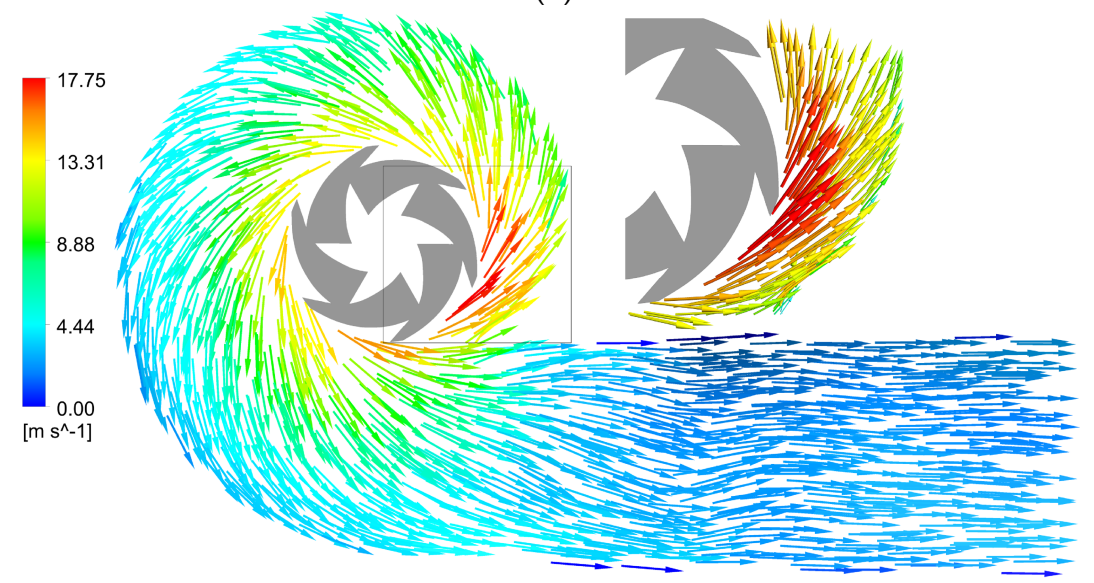

(b)

Figure 10. Velocity vector distribution at the point of $0.6 Q_{d e s}$ with different phase positions: $(\mathbf{a}) 0^{\circ}$; (b) $30^{\circ}$. 


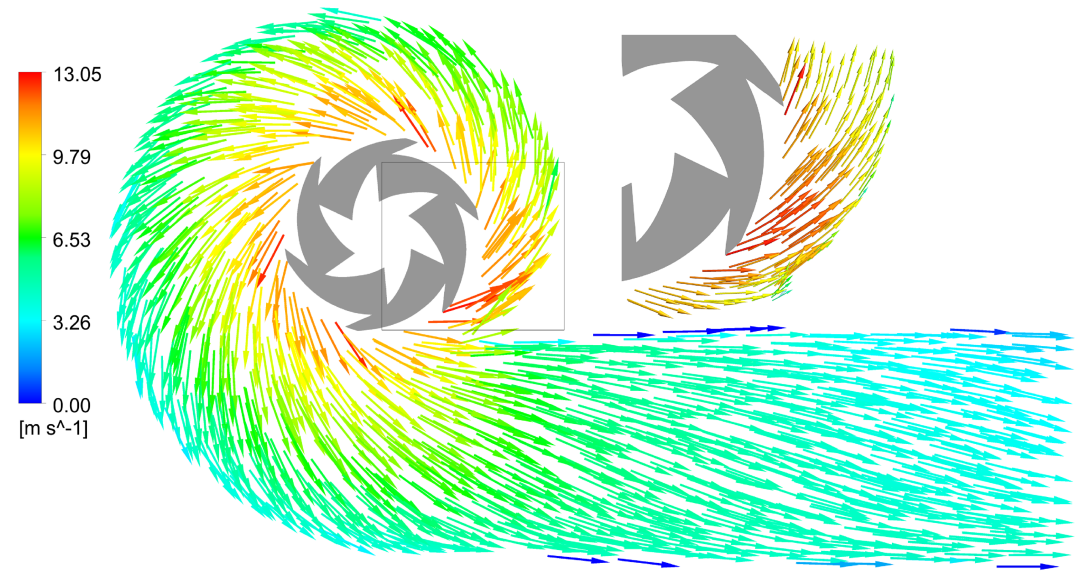

(a)

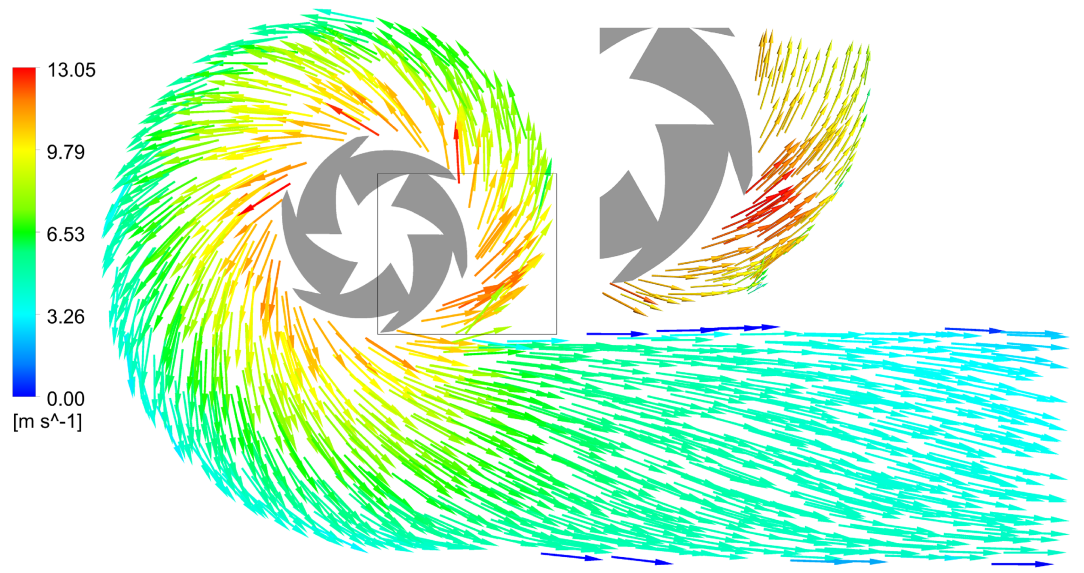

(b)

Figure 11. Velocity vector distribution at the point of $1.0 Q_{\text {des }}$ with different phase positions: $(\mathbf{a}) 0^{\circ}$; (b) $30^{\circ}$.

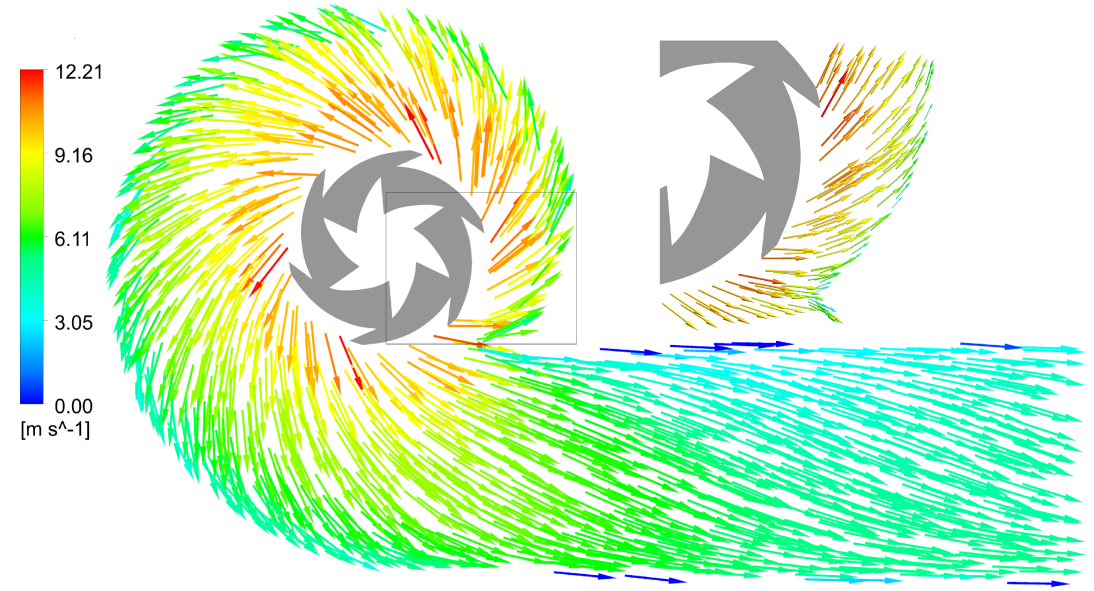

(a)

Figure 12. Cont. 


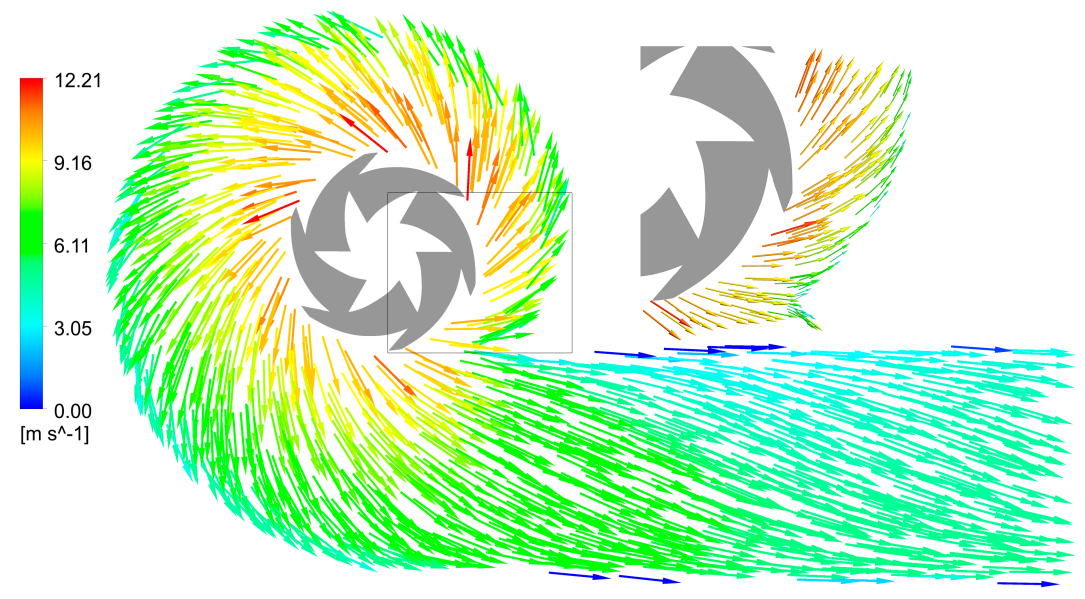

(b)

Figure 12. Velocity vector distribution at the point of $1.4 Q_{\text {des }}$ with different phase positions: $(\mathbf{a}) 0^{\circ}$; (b) $30^{\circ}$.

Primary observation shows that there are mainly two obvious differences in the comparison of flow structures. First of all, the direction of the velocity vectors near the interface between impeller and exit chamber changes a lot along the flow flux. The incidence angles for difference computation cases, which is the intersection angle of the local velocity vector and the circle tangent of the above interface, are listed in Table 6 . There are little differences when the phase position changes for the data in Table 6, but both the maximum and averages values of the incidence angle increase dramatically when the flow flux rises. Then, there are some flow structure changes near the tongue along with the rotation of impeller due to the interaction factors, but the specific difference level does not keep the same under each flow flux.

Table 6. Fluid incidence angle on the interface between impeller and exit chamber. Unit: Degree.

\begin{tabular}{ccccccc}
\hline Flow Flux & \multicolumn{2}{c}{$\mathbf{0 . 6} Q_{\text {des }}$} & \multicolumn{2}{c}{$\mathbf{1 . 0} \mathbf{Q}_{\text {des }}$} & \multicolumn{2}{c}{$\mathbf{1 . 4 Q _ { \text { des } }}$} \\
\hline Phase Position & $0^{\circ}$ & $30^{\circ}$ & $0^{\circ}$ & $30^{\circ}$ & $0^{\circ}$ & $30^{\circ}$ \\
Maximum Value & 29.33 & 24.57 & 34.4 & 36.57 & 41.81 & 43.21 \\
Average Value & 8.21 & 8.97 & 14.84 & 13.18 & 20.85 & 19.22 \\
\hline
\end{tabular}

To investigate the mechanism of the fluctuation in the efficiency at different working points, a hypothesis about the refinement of the widely applicable impeller-volute interaction theory was proposed in this work. The core idea of this hypothesis is that the fluctuation of flow parameters of centrifugal pump depends on both the structural shape of hydraulic model and flow flux. In fact, it can be judged that the affection of flow flux acts through the change of incidence angle, as can be observed in Table 6. First of all, with fixed shaft rotating speed, there is an optimal hydraulic model shape and flow flux combination for each centrifugal pump. Under these circumstances, inner fluid flow can fit well with the impeller rotation, and distribute fluid evenly with the increment of impeller's rotational angle, thus the efficiency fluctuation is relatively low. However, when the flow rate varies and disturbs the balance of the above combination, the fluctuation will increase.

At lower flow rates, the effect of hydraulic modelled shape becomes the dominating factor. As the flow rate further decreases, the impeller-volute interaction becomes stronger. This has been shown in Figure 10. There is an obvious vector distribution difference between the two phase positions. At the $0^{\circ}$ position, the velocity in the blade passage near the tongue (see the rectangular dotted box in Figure 10a) is much larger than the other region, and therefore it produces more friction, thus reducing the energy efficiency. Meanwhile, at the $30^{\circ}$ position (as shown in the rectangular dotted box of Figure 10b), the region with high flow velocity is divided into two blade passages. Therefore, 
the vector distribution becomes more uniform and the energy efficiency gets higher. Similar patterns can also be found in Figure 11, but the difference in velocity vector distribution and efficiency value between the above two phase positions becomes smaller because the flow flux rises and gets closer to the optimal rate.

However, the dominant factor changes to the flow flux when the flow rate is larger than the optimal value, and the effect of impeller-volute interaction on flow field becomes weaker. For example, in Figure 12, there is little difference between the velocity vector distributions of $0^{\circ}$ and $30^{\circ}$ positions. Due to this, the efficiency fluctuation at the $1.4 Q_{\text {des }}$ point is small.

Since the changes in velocity vector are only limited to the local blade-tongue region and they would not significantly affect the main flow features of the full field, the fluctuation of the torque applied on the impeller is larger than the head lift during the rotation. This explains why the torque fluctuation is the main factor for efficiency wave behaviors rather than the head for this research subject.

\section{Conclusions}

The present work numerically investigates the fluctuation behavior in the efficiency of a centrifugal pump with detailed and quantitative analysis. Numerical methods can provide a convenient and swift method for quantitative evaluation of the efficiency fluctuation without disturbance from the external environment factors. Some important conclusions as obtained from the current work are as follows.

(1) Centrifugal pumps show efficiency fluctuation with common dominant frequency equaling the blade passing frequency. However, the initial phase of the fluctuation curves is different for each working point.

(2) The efficiency fluctuation level is significant, and its value varies for different working points. It is relatively higher for the part-load point, however high efficiency does not necessarily result in low efficiency fluctuation.

(3) The efficiency fluctuation is caused by the impeller-volute interaction, and its effect is strong on the part-load condition. When the flow flux rises, its effect becomes less obvious and the level of efficiency fluctuation becomes relatively smaller.

(4) Compared with the head, the torque acting on the impeller provides greater influence on the efficiency fluctuation.

Taking into account many research reports on the fluctuation of pressure and fluid forces, more intensive study is still needed to understand efficiency fluctuation in centrifugal pumps. This should especially be done by using direct experimental testing and validation work with more precise measurement instruments.

Acknowledgments: The present work was supported by the Science and Technology Plan of Hunan Province, China (2014GK3150). Anonymous reviewers provided some constructive reviews, which is gratefully acknowledged.

Author Contributions: Hehui Zhang built the math model, carried out simulation computation and analyzed the data; Shengxiang Deng guided the research work and made scientific discussion; Yingjie Qu contributed experiment test; all the authors commented and wrote the article.

Conflicts of Interest: The authors declare no conflict of interest.

\section{References}

1. Zhang, H.H.; Deng, S.X.; Qu, Y.J. High working efficiency of rapid custom design. World Pumps 2016, 3, 34-36. [CrossRef]

2. Shah, S.R.; Jain, S.V.; Patel, R.N.; Lakhera, V.J. CFD for centrifugal pumps: A review of the state-of-the-art. Proc. Eng. 2013, 51, 715-720. [CrossRef]

3. Lei, T.; Zhu, B.S.; Cao, S.L.; Wang, Y.C.; Wang, B.B. Influence of prewhirl regulation by inlet guide vanes on cavitation performance of a centrifugal pump. Energies 2014, 7, 1050-1065.

4. Ding, H.; Visser, F.C.; Jiang, Y.; Furmanczyk, M. Demonstration and validation of a 3D CFD simulation tool predicting pump performance and cavitation for industrial applications. J. Fluids Eng. 2011, 133. [CrossRef] 
5. Kean, W.C.; Winoto, S.L.; Cheah, K.W. Numerical study of inlet and impeller flow structures in centrifugal pump at design and off-design points. Int. J. Fluid Mach. Syst. 2011, 4, 25-32.

6. Sten, M.; Martin, G. Experimental and numerical investigation of centrifugal pumps with asymmetric inflow conditions. J. Therm. Sci. 2015, 24, 516-525.

7. Wang, C.L.; Zeng, C.; Peng, X.Y.; Peng, H.B.; Liu, D. Numerical simulation of internal flow field and performance prediction of reversible double suction pump. J. Drain. Irrig. Mach. Eng. 2015, 33, 577-582.

8. Shi, W.D.; Chen, K.Q.; Zhang, D.S.; Xing, J. Numerical optimization and regression analysis of forward-extended double-blade sewage pump. J. Huazhong Univ. Sci. Technol. 2015, 43, 49-63.

9. González, J.; Fernández, J.; Blanco, E.; Santolaria, C. Numerical simulation of the dynamic effects due to impeller-volute interaction in a centrifugal pump. J. Fluids Eng. 2002, 124, 348-355. [CrossRef]

10. Treutz, G. Numerische Simulation der instationären Strömung in einer Kreiselpumpe. Ph.D. Thesis, University of Damstadt, Damstadt, Germany, 2002.

11. Guo, S.J.; Okamoto, H. An experimental study on the fluid forces induced by rotor-stator interaction in a centrifugal pump. Int. J. Rotating Mach. 2003, 9, 135-144. [CrossRef]

12. Cheah, K.W.; Lee, T.S.; Winoto, S.H. Unsteady fluid flow study in a centrifugal pump by CFD Method. In Proceedings of the 7th Asean ANSYS Conference, Biopolos, Singapore, 30-31 October 2008.

13. Lucius, A.; Brenner, G. Unsteady CFD simulations of a pump in part load conditions using scale-adaptive simulation. Int. J. Heat Fluid Flow 2010, 31, 1113-1118. [CrossRef]

14. Huang, S.; Yang, F.X.; Guo, J. Numerical simulation of 3D unsteady flow in centrifugal pump by dynamic mesh technique. Proc. Eng. 2013, 61, 270-275.

15. Dai, C.; Kong, F.Y.; Dong, L. Pressure fluctuation and its influencing factors in circulating water pump. J. Cent. South Univ. 2013, 20, 149-155. [CrossRef]

16. Yuan, S.Q.; Zhou, J.J.; Yuan, J.P.; Zhang, J.F.; Xu, Y.P.; Li, T. Characteristic analysis of pressure fluctuation of unsteady flow in screw-type centrifugal pump with small blade. Trans. Chin. Soc. Agric. Mach. 2012, $43,83-87$.

17. Zhang, J.F.; Wang, W.J.; Fang, Y.J.; Ye, L.T.; Yuan, S.Q. Influence of splitter blades on unsteady flow and structural dynamic characteristics of a molten salt centrifugal pump. J. Vib. Shock 2014, 33, 37-41.

18. Barrio, R.; Parrondo, J.; Blanco, E. Numerical analysis of the unsteady flow in the near-tongue region in a volute-type centrifugal pump for different operating points. Comput. Fluids 2010, 39, 859-870. [CrossRef]

19. Pei, J.; Yuan, S.Q.; Li, X.J.; Yuan, J.P. Numerical prediction of 3-D periodic flow unsteadiness in a centrifugal pump under part-load condition. J. Hydrodyn. 2014, 26, 257-263. [CrossRef]

20. Liu, H.L.; Liu, D.X.; Wang, Y.; Wu, X.F.; Wang, J.; Du, H. Experimental investigation and numerical analysis of unsteady attached sheet cavitation flows in a centrifugal pump. J. Hydrodyn. 2013, 25, 370-378. [CrossRef]

21. ANSYS, Inc. ANSYS Help System; ANSYS, Inc.: Canonsburg, PA, USA, 2011.

22. Menter, F.R. Two-equation eddy-visocity turbulence models for engineering applications. AIAA J. 1994, 32, 1598-1605. [CrossRef]

23. The International Standards Organization for Standardization. ISO 9906-2012. Rotodynamic Pumps-Hydraulic Performance Acceptance Tests-Grades 1, 2 and 3, 2nd ed.; The International Standards Organization for Standardization: Geneva, Switzerland, 2015.

24. Kim, J.H.; Oh, K.T.; Pyun, K.B.; Kim, C.K.; Choi, Y.S. Design optimization of a centrifugal pump impeller and volute using computational fluid dynamics. In Proceedings of the 26th IAHR Symposium on Hydraulic Machinery and Systems, Beijing, China, 19-23 August 2012.

25. Zhao, W.G.; Sheng, J.P.; Yang, J.H.; Song, Q.C. Optimization design and experiment of centrifugal pump based on CFD. Trans. Chin. Soc. Agric. Mach. 2015, 31, 125-131.

(c) 2017 by the authors. Licensee MDPI, Basel, Switzerland. This article is an open access article distributed under the terms and conditions of the Creative Commons Attribution (CC BY) license (http:/ / creativecommons.org/licenses/by/4.0/). 